\title{
Study of the Possibility of Reducing the Slow Fluctuations of the Reactivity and Thermal Power of the IBR-2M Reactor
}

\author{
Yuri Pepelyshev ${ }^{1, \star}$ and Tsolmon Tsogtsaikhan ${ }^{1,2, \star \star}$ \\ ${ }^{1}$ Joint Institute for Nuclear Research, Dubna, Russia \\ ${ }^{2}$ Institute of Physics and Technology, Ulaanbaatar, Mongolia
}

\begin{abstract}
This paper presents an artificial neural network method for long-term prediction of the thermal dynamic parameters of the IBR-2M reactor. Attention is focused mainly on the prediction of the temperature and sodium flow at the entry into the core as well as the thermal power. It is shown that the prediction makes it possible to reduce by a factor of 3 the influence of slow fluctuations of reactivity on the power and thereby reduce the operational requirements for the automatic power stabilization system.
\end{abstract}

\section{Introduction}

The random variations of the thermodynamic parameters of the primary cooling circuit of the sodium cooling system of the core in the IBR-2M reactor in normal operation cause large fluctuations in reactivity, which lead to changes in pulse energy and power, respectively. The automatic stabilization system compensates for these changes and maintains the power at a prescribed level. The concept of power stabilization is based on the knowledge of the energy of the running pulse and of some number of preceding pulses. The value of the previous information decreases in time. It is clear that the knowledge of the energy of pulses subsequent to the current power pulse or subsequent changes in reactivity can improve the stabilization and facilitate at high-load an operating regime securing the stabilization of the system itself. The aim of the present work is to investigate the possibility of predicting fluctuations of the thermodynamic parameters of the cooling system of the IBR-2M reactor core and the associated reactivity fluctuations. The possibility of reducing the reactivity fluctuations according to the prediction is analyzed.

\section{Experimental Data and Method}

The IBR-2M reactor is located at the Joint Institute for Nuclear Research (Dubna, Russia) and operates with the design power of $2 \mathrm{MW}$. The IBR-2M core capacity is 69 fuel elements, which are sleeve-like $\mathrm{PuO}_{2}$ pellets. The coolant is liquid sodium, pumped through the emergency protection blocks by two induction pumps. There are two blades rotating with different speeds past one of the core faces. They are the main moving reflector and the auxiliary moving reflector of the reactivity modulator. The reactivity level is adjusted by the control and protection system elements, which are

\footnotetext{
$\star$ e-mail: pepel@nf.jinr.ru

$\star \star$ e-mail: tsolmonuc@gmail.com
} 
movable tungsten blocks in an array of fixed steel reflectors. The reactor core is surrounded by water moderators enabling the creation of thermal neutrons for users.

IBR-2M operates in cycles: two weeks at $2 \mathrm{MW}$ followed by shutdown for one week (power close to zero). The cycles are repeated periodically. The initial time series comprises an array of successive values of the sodium flow $G_{\Sigma}$ and the sodium temperature $T_{i n}$ at the inlet into the core, the thermal power $W_{T}$, and the ratio $W_{T} / G_{\Sigma}$ proportional to the heating. The sodium temperature at the core inlet and outlet $T_{\text {out }}$, the total sodium flow $G_{\Sigma}=G_{A}+G_{B}$ in the circuits $\mathrm{A}\left(G_{A}\right)$ and B $\left(G_{B}\right)$ in the primary cooling circuit were measured during several reactor cycles in 2014. The data survey period was 0.1 sec for all parameters. The length of the time series in the measurements reached $\sim 6 \times 10^{6}$ values. The thermal power was estimated as $W_{T}=G_{\Sigma}(\Delta T-0.252) / 3.15$, where $\Delta T=\left(T_{\text {out }}-T_{\text {in }}\right)$ is the heating of the sodium in the reactor core. This sample of the parameters values are the basis for prediction. A neural net model for predicting the course of the time series was chosen as the prediction system [1-3].

\section{Results}

The analysis showed that a slow change (at the level of daily fluctuations) in the main parameters of the primary cooling circuit of the cooling system of the IBR-2M core can be predicted with satisfactory accuracy for reactor operation up to 5-7 days (Fig. 1).

The changes in reactivity $\rho_{T}(t)$ and sodium flow $\rho_{G_{\Sigma}}(t)$ accompanying a deviation of the temperature from the average values $G_{0}$ and $T_{0}$, respectively, are characterized in terms of the ratios $\Delta K / \Delta G_{\Sigma}$ and $\Delta K / \Delta T$ which denote the flow and temperature coefficients of reactivity, respectively [4-6]. The predicted reactivity $\Delta \rho_{\Sigma}(t)$ is a previously known process, which, in principle, can be excluded from the overall change in reactivity (Fig. 2). The method for eliminating it may be different, for example, the introduction of a reactivity $\Delta \rho_{\Sigma}(t)$ of the opposite sign using an addition control rod. In this case, this is not fundamental because only the possibility of reactivity compensation and not the method of its implementation is under study.

\section{Conclusion}

We presented predictions, within five percent accuracy, obtained by means of nonlinear auto regression neural net software concerning changes in the thermodynamic parameters of the IBR-2M primary cooling circuit (liquid-sodium). The input of the method uses values of measured corresponding quantities at previous primary cycles.

On the basis of the predicted parameters we have determined the needed compensating reactivity. Up to now this was hardwired in the automatic power stabilization system. Although we predict only the slow-component of the compensation, the residual (that the stabilization system is left to determine on its own) is approximately three times smaller.

The prediction eliminates stabilization system wear out, thereby increasing the reliability and safety of the reactor. Our future work will be directed to the study of the reliability of the software under (unexpected) shocks.

\section{References}

[1] G. Ososkov, Yu. Pepelyshev, and Ts. Tsogtsaikhan, European Physical Journal 108, 02036 (2016)

[2] Yu. Pepelyshev, Ts. Tsogtsaikhan, and G. Ososkov, Physics of Particles and Nuclei Letters 13, 704-707 (2016) 


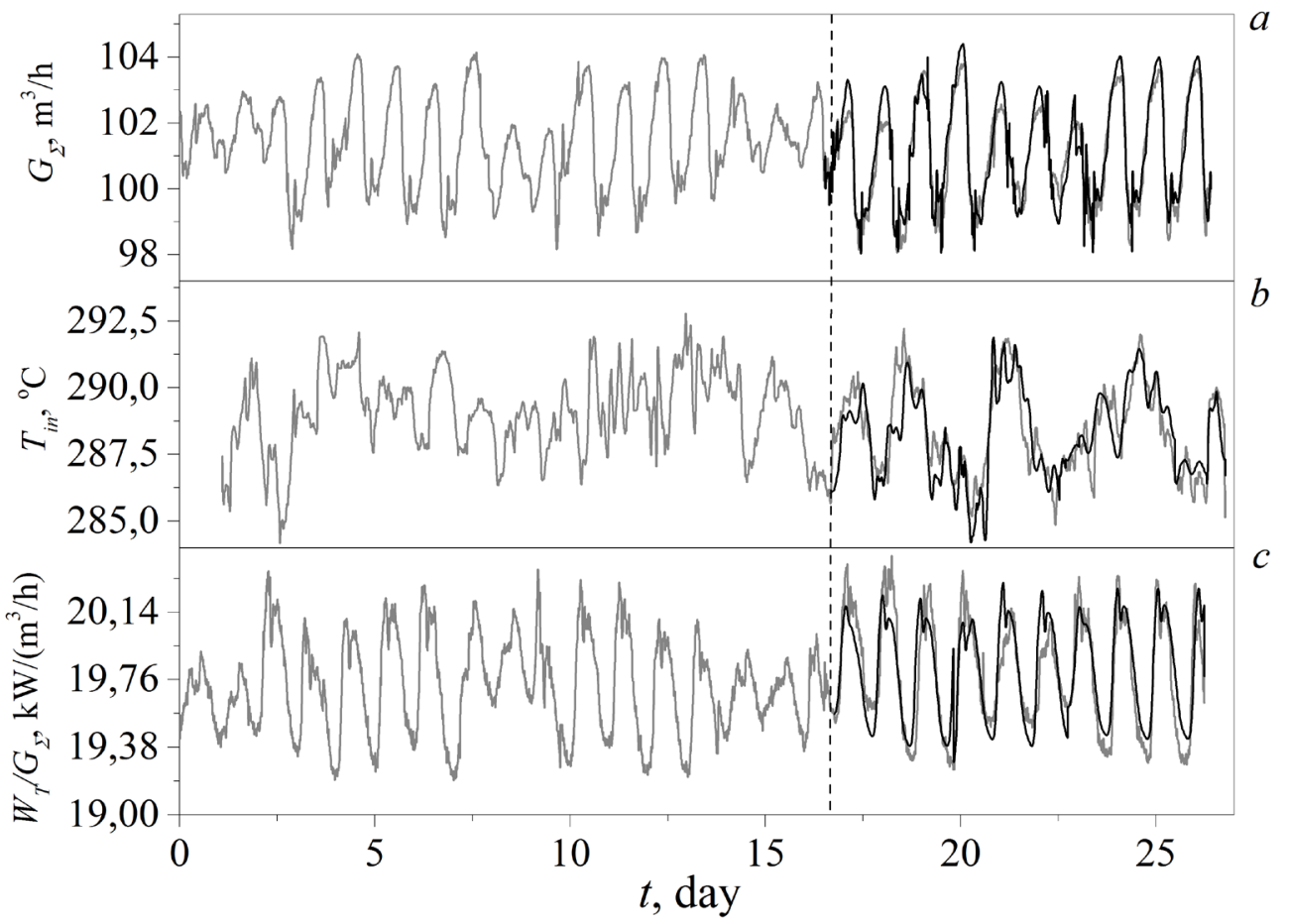

Figure 1. Change in the sodium flow $G_{\Sigma}$ (a), sodium temperature at the inlet into the core $T_{\text {in }}(\mathrm{b}), W_{T} / G_{\Sigma}$ proportional to the heating (c) versus the operating time of the reactor $(t)$. The experimental data (grey lines) extend over the whole variation range of the operating time. Predictions (black lines) start at the broken separation line and are in qualitative agreement with the corresponding measured data set at a given time

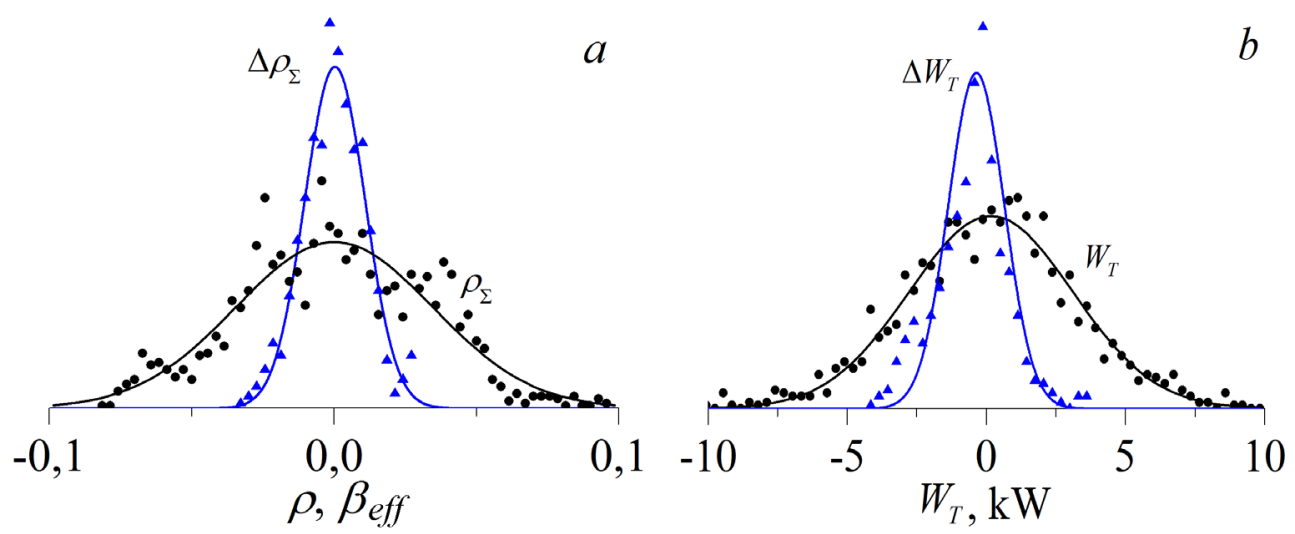

Figure 2. Distribution of the reactivity fluctuations before $\left(\rho_{\Sigma}\right)$ and after $\left(\Delta \rho_{\Sigma}\right)$ the prediction (a) and thermal power before $\left(W_{T}\right)$ and after $\left(\Delta W_{T}\right)$ the prediction (b) 
[3] Yu. Pepelyshev and Ts. Tsogtsaikhan, Atomic Energy 121, 173-178 (2017)

[4] Yu. Pepelyshev and Ts. Tsogtsaikhan, Preprint JINR R13-2014-61, 1-16 (2014)

[5] V. Anan'ev, A. Vinogradov, A. Dolgikh, et al., Preprint JINR R13-2012-41, 1-21 (2012)

[6] V. Anan'ev, A. Vinogradov, A. Dolgikh, et al., Preprint JINR R13-2012-42, 1-24 (2012) 